\title{
Novel Perceived Stress and Life Events Precede Flares of Inflammatory Bowel Disease: A Prospective 12-Month Follow-Up Study
}

\author{
Dion S. J. Wintjens, ${ }^{a, b}$ Marin J. de Jong, ${ }^{a}$ Andrea E. van der Meulen-de Jong, ${ }^{c}$ \\ Mariëlle J. Romberg-Camps, ${ }^{d}$ Marco C. Becx, ${ }^{e}$ Jeroen P. Maljaars, ${ }^{\mathrm{c}}$ \\ Ad A. van Bodegraven, ${ }^{d}$ Nofel Mahmmod, ${ }^{e}$ Tineke Markus, ${ }^{f}$ Jeoffrey Haans, ${ }^{a}$ \\ Ad A.M. Masclee, ${ }^{a, b}$ Bjorn Winkens, ${ }^{g}$ Daisy M. A. E. Jonkers, ${ }^{a, b}$ Marie J. Pierik ${ }^{a, b}$
}

aDepartment of Internal Medicine, Division of Gastroenterology and Hepatology, Maastricht University Medical Centre, Maastricht, The Netherlands bNUTRIM School for Nutrition and Translational Research in Metabolism, Maastricht University Medical Centre, Maastricht, The Netherlands 'Department of Gastroenterology and Hepatology, Leiden University Medical Centre, Leiden, The Netherlands dDepartment of Gastroenterology and Hepatology, Zuyderland Medical Centre, Sittard-Geleen, The Netherlands eDepartment of Gastroenterology and Hepatology, St. Antonius Hospital, Nieuwegein, The Netherlands ${ }^{\mathrm{f} C C U V N}$, Dutch IBD Patients Organization, Woerden, The Netherlands ${ }^{9}$ Department of Methodology and Statistics, Care and Public Health Research Institute (CAPHRI), Maastricht, The Netherlands

Corresponding author: Dion Wintjens, MD, Division of Gastroenterology and Hepatology, Department of Internal Medicine, NUTRIM, School of Nutrition and Translational Research in Metabolism, Maastricht University Medical Centre, Postbox 5800, 6202 AZ, Maastricht, The Netherlands. Tel: 0031-43-3875021; Fax: 0031-43-3875006; Email: d.wintjens@maastrichtuniversity.nl

Conference: $25^{\text {th }}$ UEG Week, Barcelona, 2017.

\begin{abstract}
Background and Aims: Inflammatory bowel disease [IBD] is characterized by recurrent disease flares. The impact of psychosocial wellbeing on the occurrence of flares is unclear. In this prospective study, we aimed to evaluate the association between patient-reported psychosocial wellbeing and disease flares using continuous monitoring.

Methods: Consecutive IBD patients were recruited from the mylBDcoach telemedicine study cohort. Over 12 months, participants reported on disease activity together with anxiety, depression, fatigue, perceived stress and life events every 1-3 months. Flares were defined using a combination of clinical disease activity and additional measurements. Generalized estimating equation models were used to assess associations between psychosocial wellbeing and flares over time. The influences of both the presence of psychosocial symptoms in general as well as novel psychosocial symptoms were analysed. Results: In total, 417 patients were included. Forty-nine patients [11.8\%] experienced a flare during the study period. The occurrence of life events in the preceding 3 months was positively associated with flares (odds ratio $[\mathrm{OR}]=1.81 ; 95 \%$ confidence interval $[\mathrm{Cl}]=1.04-3.17$ ), while the presence of anxiety, depression, fatigue and perceived stress in general was not. However, novel perceived stress $[\mathrm{OR}=2.92 ; 95 \% \mathrm{Cl}=1.44-5.90]$ was associated with flares.

Conclusions: The occurrence of life events and novel perceived stress are associated with disease flares in the next 3 months, while the presence of perceived stress in general is not. These findings underline the importance of continuous personalized monitoring of IBD patients and may contribute to the prevention of disease flares.
\end{abstract}

Key Words: IBD; telemedicine; psychosocial wellbeing 


\section{Introduction}

Inflammatory bowel disease [IBD], comprising Crohn's disease [CD] and ulcerative colitis [UC], is a chronic and disabling disease characterized by recurrent periods of disease activity. These flares warrant escalation of medical treatment and often require hospitalizations or surgery. Recurrent flares may also lead to irreversible bowel damage and disease progression [e.g. the occurrence of complications such as fistulas and stenosis]. ${ }^{1,2}$ Although biochemical markers are available for early detection of disease activity, ${ }^{3}$ identification of the factors contributing to the emergence of flares might be an even more interesting approach to improve the outcome of IBD.

Regarding potentially flare-inducing factors, psychosocial wellbeing of patients is of interest. While not systematically monitored by healthcare professionals in routine care, a high prevalence of psychological disorders such as anxiety and depression is reported in the IBD population. ${ }^{4,5}$ This may be a consequence of the chronic disease itself, but it is also hypothesized that impaired psychosocial wellbeing can negatively impact gut health through multiple immuneneuroendocrine brain-gut interaction pathways. ${ }^{6,7}$

A large number of studies have investigated the association between psychosocial wellbeing and disease activity in IBD, although many are limited by their cross-sectional design, thereby potentially neglecting the aforementioned bi-directionality of the brain-gut axis. When taking into account only longitudinal studies, contradictory results have been reported regarding the effects of anxiety and depression on flares. ${ }^{8}$ Perceived stress and life events have been studied more extensively and it has been reported that a positive association exists between flares and perceived stress in most longitudinal studies. ${ }^{9-14}$ In many studies, however, careful interpretation is advocated due to low sample size and the sole use of clinical disease activity indices to define flares, because these indices are poor predictors of mucosal inflammation. ${ }^{15}$ Also, perceived stress may affect intestinal motility and may magnify visceral sensitivity, leading to worsening of abdominal symptoms in the absence of inflammation. ${ }^{16,17}$ It remains debatable to what extent mood disorders and perceived stress are associated with disease flares. For instance, because both depression and perceived stress can be present for longer periods, ${ }^{13,18}$ it may be relevant to focus on within-subject changes in psychosocial wellbeing rather than to analyse only the presence of impaired psychosocial wellbeing preceding flares, which has, to our knowledge, not been done before.

Recently, de Jong et al. showed that continuous monitoring at home with the telemedicine tool 'myIBDcoach' is safe and even reduced outpatient visits and hospital admissions. ${ }^{19}$ myIBDcoach continuously monitors patient-reported disease activity and psychosocial wellbeing through questionnaires. This method of data collection may provide important insights into psychosocial wellbeing over time. In this prospective study, we aimed to evaluate which domains of psychosocial wellbeing, when impaired, were associated with future disease flares based on data from the myIBDcoach trial.

\section{Materials and methods}

\subsection{Design and procedures}

All data used in this study were derived from the myIBDcoach study cohort. The design of this randomized controlled telemedicine study has previously been described in detail. ${ }^{19}$ In short, IBD patients were included in four hospitals in the Netherlands [i.e. two academic hospitals, Maastricht University Medical Centre and Leiden University Medical Centre, and two regional hospitals, Zuyderland Medical Centre Sittard and St. Antonius Hospital Nieuwegein]. Patients between 18 and 75 years of age who had a histopathologically corroborated diagnosis of IBD were eligible for inclusion. Exclusion criteria comprised insufficient knowledge of the Dutch language, lack of internet access by computer, tablet or smartphone, or a hospital admission within 2 weeks prior to inclusion. Also, patients with an ileoanal pouch or ileorectal anastomosis were excluded. After inclusion, patients were randomized in a 1:1 ratio to standard care [control group] or to care through myIBDcoach [intervention group] and followed for at least 12 months. Details of the myIBDcoach telemedicine system have been described elsewhere. ${ }^{20}$ In brief, myIBDcoach is a secure webpage which is accessible through computer, tablet or smartphone. The system provides monthly monitoring modules, including questions regarding disease activity, use of medication, treatment adherence, smoking status and psychosocial wellbeing. When patients achieved sustained remission [i.e. low clinical activity for three consecutive months], the system was allowed to complete the monitoring module once every 3 months. When predefined thresholds, for instance on clinical disease activity, were exceeded, a healthcare provider of the local team contacted the patient for further assessment. The study was approved by the Medical Research Ethics Committee of the Maastricht University Medical Centre, being applicable to all participating centres. This trial was registered at ClinicalTrials.gov [NCT02173002].

For the present study, we included all patients who received care through myIBDcoach [i.e. the intervention arm of the randomized study]. Patients without a single period of documented remission during follow-up were excluded because this period of remission was required to analyse the association between the development of disease flares and preceding psychosocial wellbeing. Detailed baseline data at the time of inclusion in myIBDcoach [i.e. patient characteristics, disease phenotype, disease duration and previous treatments] were collected by scrutinizing patient files using standardized registration forms. During the 12 months of follow-up, questionnaires on disease activity [MIAH-questionnaire $\left.{ }^{21}\right]$ and psychosocial wellbeing [i.e. anxiety, depression, fatigue, perceived stress and life-events] were completed every 1-3 months.

\subsection{Definitions}

In line with previous analyses based on the myIBDcoach tool, flares were defined as clinical symptoms [i.e. positive score on the MIAHquestionnaire or symptoms during outpatient visits] indicative of disease activity in combination with at least one of the following: concurrent calprotectin $>250 \mu \mathrm{g} / \mathrm{g}$ in the stool or active disease determined by endoscopy, magnetic resonance imaging or computed tomography. In daily practice, in case of clinically severe symptoms suggestive for IBD disease activity, the treating physician occasionally judged these symptoms to be evident enough to adjust therapy. Therefore, to capture all flares, also clinical episodes were defined as flares if symptoms suggestive of IBD disease activity resulted in a dose escalation or initiation of a new drug to induce remission.

Psychosocial parameters were assessed through a patient-reported questionnaire containing one question for each psychosocial domain of interest [Table 1]. For anxiety, depression and fatigue, questions could be answered on a five-point Likert-scale [i.e. 'seldom or never', 'occasionally', 'regularly', 'often', or 'always or almost always']. Cutoffs were determined based on the smallest Euclidian distances compared to validated questionnaires [i.e. HADS-A,,$^{22}$ HADS-D ${ }^{22}$ and VVS $^{23}$. The performance of the questionnaire is shown in Table 1. For perceived stress, we used a visual analogue scale on which a patient could report on their current stress level [range from 1 to 10] in which a higher score reflects higher stress levels. This scale showed 
Table 1. Overview of psychosocial questionnaire used in the mylBDcoach telemedicine tool

\begin{tabular}{|c|c|c|c|c|}
\hline Domain & Question & Scale & Cut-off & $\begin{array}{l}\text { Performance vs validated } \\
\text { questionnaire [sens/spec] }\end{array}$ \\
\hline Anxiety & In the past two weeks I ... felt anxious. & Likert $[1-5]$ & 2 [occasionally] & HADS-A ${ }^{22}[64 / 82]$ \\
\hline Depression & In the past two weeks I ... felt miserable or depressed. & Likert $[1-5]$ & 3 [frequently] & HADS-D $^{22}[73 / 93]$ \\
\hline Fatigue & In the past two weeks I ... felt tired. & Likert $[1-5]$ & 3 [frequently] & $\mathrm{VVV}^{23}[90 / 100]$ \\
\hline Perceived stress & $\begin{array}{l}\text { Rate your current stress level on a scale from } 1 \text { to } 10 \text {, what } \\
\text { number would you give? }\end{array}$ & VAS [1-10] & NA & $\operatorname{PSS}^{24}[R=0.654]$ \\
\hline Life events & $\begin{array}{l}\text { Did you recently experience any important or drastic events } \\
\text { which influenced your feelings? }\end{array}$ & Dichotomous & NA & NA \\
\hline
\end{tabular}

VAS, visual analogue scale; NA, not applicable; sens, sensitivity; spec, specificity; HADS-D, Hospital Anxiety and Depression Scale - Depression; HADS-A, Hospital Anxiety and Depression Scale - Anxiety; VVV, Verkorte VermoeidheidVragenlijst [translated: Short Fatigue Questionnaire]; PSS, Perceived Stress Scale.

a moderate but significant correlation with the Cohen's Perceived Stress Scale [PSS; $r=0.654] .{ }^{24}$ However, note that the PSS is based on perceived stress during the previous month, while our scale measures current stress levels. A cut-off point of 4, based on expert opinion of our team of gastroenterologists and our population's average, was used to differentiate low vs high perceived stress levels. The occurrence of life events was scored in a binary approach [i.e. yes/no]. The underlying reason for the life event was answered as an open-ended question and was therefore neglected in the analysis.

\subsection{Statistical analysis}

Baseline characteristics are presented as means with corresponding standard deviations [SD] for numerical variables with a normal distribution, as medians with corresponding interquartile ranges [IQR] for numerical variables without a normal distribution, and as number of patients [\%] for categorical variables. For comparison between groups, the independent-samples $t$-test and chi-square test or Fisher's exact test, when appropriate, were used for numerical and categorical variables, respectively.

Generalized estimating equation [GEE] models were used to identify associations between psychosocial wellbeing and the subsequent development of flares over time. This method is used to account for repeated measures within the same patient, where the covariance pattern was unstructured. Because it was unknown how long a patient was in remission when entering the study, the first 3 months of all patients were ignored in the analyses. Next, each month of follow-up was considered either as positive or negative with respect to flares, according to the aforementioned definition. Also, patient-reported psychosocial wellbeing was assessed with intervals of 1 month using the aforementioned cut-offs. When active disease was present at baseline or when a flare occurred during follow-up, the subsequent 6 months were excluded from the analysis to correct for a potential effect of disease activity and the subsequent remission-induction therapy on the psychosocial wellbeing of a patient. This can be considered as a wash-out period for both gastrointestinal inflammation and psychosocial imbalance caused by the preceding flare. Because patients could choose to fill in the questionnaires either once every month or once every 3 months, all models estimate the effect of psychosocial wellbeing on disease activity in the next quarter.

In the first GEE model, the association between flares and the presence of psychosocial symptoms in general was assessed. Predefined cut-offs were used to indicate the presence or absence of all psychosocial factors available in myIBDcoach, regardless of the prior psychosocial wellbeing of the patient. In the second GEE model, the association between flares and novel psychosocial symptoms was assessed. Here, differences in psychosocial parameters over time were determined to separate novel symptoms from pre-existing symptoms. At each time point the alteration in psychosocial parameters was regarded as positive when this change exceeded the cut-off values of these parameters (e.g. from 'seldom or never feeling depressed' [i.e. score 1] to 'often feeling depressed' [i.e. score 4]) in the 3 months preceding this time point. Life events were only analysed in the first model, because life events are 'novel' by definition and therefore the second model is not applicable. To ensure a prospective association between the psychosocial parameters and subsequent flares, psychosocial parameters were not taken into account as predictors when positive at the same time point as the flare. For example, when a patient experienced a flare between time points 5 and 6 , the latter was regarded as the 'time point of flare' and psychosocial scores from time points 3 to 5 were used in the model as explanatory variables.

All results were analysed using a multivariable model including gender, disease phenotype, disease duration at baseline and smoking status at baseline to correct for their potential confounding effect on disease flares. Although our study was underpowered for additional confounders, medication use at baseline was added to the model as a sensitivity analysis. All analyses were conducted using a significance level of 0.05 . Two-sided $p$-values $\leq 0.05$ were considered as statistically significant.

\section{Results}

In total, 465 patients were allocated to the myIBDcoach intervention. ${ }^{19}$ Of these, nine never started using the application and 18 were lost to follow-up. After exclusion of these patients and those with IBD unclassified [IBD-U, $n=3$ ], those without any completed questionnaire $[n=11]$ and those without a single period of remission $[n=7], 417$ patients were eligible for this study. Of these, 49 [11.8\%] developed a flare during follow-up [i.e. change from inactive to active disease]. Two of these patients developed a second flare after remission was re-obtained. Flares were defined by clinical symptoms in combination with an elevated faecal calprotectin, active disease on endoscopy or imaging, and adjustment of therapy by a gastroenterologist in 14 [27.4\%], 21 [41.2\%] and 16 [31.4\%] cases, respectively. Baseline characteristics of these patients [i.e. relapsers] compared to those not developing a flare during follow-up [i.e. non-relapsers] are presented in Table 2. No significant differences in baseline characteristics were found between relapsers and non-relapsers, except for a greater proportion of smokers in the latter group [ $p=0.005]$. The average time from inclusion to their first disease flare for relapsers was $6.4[\mathrm{SD}=2.6]$ months. 
Table 2. Baseline characteristics, stratified by occurrence of disease flares

\begin{tabular}{|c|c|c|c|}
\hline & $\begin{array}{l}\text { Relapsers } \\
{[n=49]}\end{array}$ & $\begin{array}{l}\text { Non-relapsers } \\
{[n=368]}\end{array}$ & $p$-value \\
\hline Crohn's disease, $n[\%]$ & $26[53.1]$ & $222[60.3]$ & 0.331 \\
\hline Male, $n[\%]$ & 15 [30.6] & 158 [42.9] & 0.100 \\
\hline \multicolumn{4}{|l|}{ Montreal at diagnosis ${ }^{a}$} \\
\hline Age [CD and UC] & & & 0.600 \\
\hline $\mathrm{A} 1, n[\%]$ & 8 [16.3] & $60[16.3]$ & \\
\hline $\mathrm{A} 2, n[\%]$ & $26[53.1]$ & 219 [59.5] & \\
\hline $\mathrm{A} 3, n[\%]$ & 15 [30.6] & 89 [24.2] & \\
\hline Disease location $[\mathrm{CD}]$ & & & 0.741 \\
\hline $\mathrm{L} 1, n[\%]$ & 7 [26.9] & 73 [32.9] & \\
\hline $\mathrm{L} 2, n[\%]$ & $6[23.1]$ & $55[24.8]$ & \\
\hline $\mathrm{L} 3, n[\%]$ & $13[50.0]$ & 94 [42.3] & \\
\hline Disease behaviour [CD] & & & 0.958 \\
\hline $\mathrm{B} 1[\mathrm{CD}], n[\%]$ & 16 [61.6] & 134 [60.6] & \\
\hline $\mathrm{B} 2[\mathrm{CD}], n[\%]$ & 7 [26.9] & $57[25.8]$ & \\
\hline $\mathrm{B} 3[\mathrm{CD}], n[\%]$ & $3[11.5]$ & 30 [13.6] & \\
\hline Disease extent $[\mathrm{UC}]$ & & & 0.768 \\
\hline $\mathrm{E} 1[\mathrm{UC}], n[\%]$ & $2[8.7]$ & 19 [12.8] & \\
\hline $\mathrm{E} 2[\mathrm{UC}], n[\%]$ & 10 [43.5] & 69 [46.3] & \\
\hline $\mathrm{E} 3[\mathrm{UC}], n[\%]$ & $11[47.8]$ & $61[40.9]$ & \\
\hline Age at baseline [years], mean $[\mathrm{SD}]$ & $46.08[15.24]$ & $43.95[13.71]$ & 0.313 \\
\hline Disease duration at baseline [years], median [IQR] & $4.65[3.38-17.8]$ & $10.8[5.2-20.8]$ & 0.172 \\
\hline Active disease at baseline, $n[\%]$ & $4[8.2]$ & $28[7.6]$ & 0.908 \\
\hline Medication use at baseline & & & 0.434 \\
\hline No medication/5-ASA, $n[\%]$ & $15[30.6]$ & 142 [39.3] & \\
\hline Immunomodulators, $n$ [\%] & 16 [32.7] & $93[25.8]$ & \\
\hline Biologics, $n[\%]$ & 18 [36.7] & $126[34.9]$ & \\
\hline Current smoking at baseline, $n[\%]$ & $1[2.0]$ & $65[17.7]$ & $0.005 *$ \\
\hline History of IBD-related surgery at baseline, $n[\%]$ & $5[10.2]$ & $41[11.1]$ & 0.470 \\
\hline
\end{tabular}

n, number of patients; CD, Crohn's disease; UC, ulcerative colitis; SD, standard deviation; IQR, interquartile range; 5-ASA, 5-aminosalicylic acid. Age was defined as below 17 years [A1], between 17 and 40 years [A2] and above 40 years [A3]. Disease location of CD was defined as ileal involvement [L1], exclusive colonic involvement [L2] and ileocolonic involvement [L3]. Disease behaviour of CD was defined as non-stricturing/non-penetrating [B1], stricturing [B2], penetrating [B3]. Disease extent of UC was defined as ulcerative proctitis [E1], left-sided UC [E2] and extensive UC [E3].

* $p<0.05$.

${ }^{a}$ According to Montreal Classification. ${ }^{36}$

Regarding the presence of psychosocial symptoms in general, only the occurrence of life events (odds ratio $[\mathrm{OR}]=1.81,95 \%$ confidence interval $[95 \% \mathrm{CI}]=1.04-3.17)$ was associated with flare development in the next 3 months [Table 3]. Neither anxiety $[\mathrm{OR}=0.73,95 \% \mathrm{CI}=0.38-1.39]$, depression $[\mathrm{OR}=1.33,95 \%$ $\mathrm{CI}=0.70-2.55]$, fatigue $[\mathrm{OR}=1.91,95 \% \mathrm{CI}=0.98-3.73]$ nor perceived stress $[\mathrm{OR}=1.09,95 \% \mathrm{CI}=0.62-1.91]$ were statistically significantly associated with flare development. Regarding novel psychosocial symptoms [i.e. alteration exceeding cut-off], only perceived stress $[\mathrm{OR}=2.92,95 \% \mathrm{CI}=1.44-5.90]$ was associated with subsequent flares [Table 3], independent of the presence of other psychosocial factors in general. No statistically significant association with subsequent flares was found in this second GEE model for anxiety $[\mathrm{OR}=1.12,95 \% \mathrm{CI}=0.50-2.51]$, depression $[\mathrm{OR}=1.28$, $95 \% \mathrm{CI}=0.54-3.07]$ and fatigue $[\mathrm{OR}=1.58,95 \% \mathrm{CI}=0.79-3.15]$. All results were adjusted for gender, disease phenotype, disease duration and smoking status. When results were analysed for CD and UC separately, the association between flares and both life events and perceived stress tended to be stronger in CD. However, due to the small number of relapses, the study was not powered to analyse these associations and the results should be interpreted with care.

In a first sensitivity analysis, medication use at baseline was added to the model. By applying this, all results remained similar. As a second sensitivity analysis, different cut-offs of the questions
Table 3. Association between both the presence of psychosocial symptoms in general [GEE model 1] as well as novel psychosocial symptoms [GEE model 2] and disease flares

\begin{tabular}{|c|c|c|c|c|}
\hline & \multicolumn{2}{|c|}{$\begin{array}{l}\text { GEE model 1: pres- } \\
\text { ence of psychosocial } \\
\text { symptoms in general }\end{array}$} & \multicolumn{2}{|c|}{$\begin{array}{l}\text { GEE model 2: } \\
\text { novel psychosocial } \\
\text { symptoms }\end{array}$} \\
\hline & OR & $95 \% \mathrm{CI}$ & OR & $95 \% \mathrm{CI}$ \\
\hline Anxiety & 0.73 & $0.38-1.39$ & 1.12 & $0.50-2.51$ \\
\hline Depression & 1.33 & $0.70-2.55$ & 1.28 & $0.54-3.07$ \\
\hline Fatigue & 1.91 & $0.98-3.73$ & 1.58 & $0.79-3.15$ \\
\hline Perceived stress & 1.09 & $0.62-1.91$ & 2.92 & $1.44-5.90^{* *}$ \\
\hline Life events & 1.81 & $1.04-3.17 *$ & NA & NA \\
\hline
\end{tabular}

OR, odds ratio; 95\% CI, 95\% confidence interval; NA, not applicable. All analyses were adjusted for gender, disease phenotype, disease duration at baseline, and smoking status at baseline.

" $p<0.05 ; * * p<0.01$.

regarding depression, anxiety, fatigue and perceived stress were used. For depression, anxiety and fatigue, neither higher nor lower cutoffs changed the results. For novel perceived stress, a difference was observed. Where cut-offs higher than 4 were chosen, perceived stress was no longer statistically significant [cut-off $5:$ OR $=1.52,95 \%$ 
$\mathrm{CI}=0.67-3.45]$, while cut-offs below 4 remained statistically significant [cut-off $3:$ OR $=2.24,95 \% \mathrm{CI}=1.02-4.90$ ].

\section{Discussion}

In this prospective continuous telemedicine monitoring study, we evaluated the effect of psychosocial wellbeing on subsequent disease flares. A positive association was found between both life events as well as perceived stress and disease flares. We thus identified a relevant difference between the presence of perceived stress in general, irrespective of changes over time, and novel perceived stress. Only the latter was prospectively associated with occurrence of disease flares in the next 3 months. Anxiety, depression and fatigue were not associated with disease flares.

The observed association between disease activity and perceived stress has previously been described. However, the power of many studies was limited due to methodological flaws such as crosssectional analyses, small sample size and the lack of objective measurements of disease activity. ${ }^{25}$ Because IBD patients often experience irritable bowel syndrome-like complaints, the sole use of clinical disease activity indices to define flares may give an overestimation of relapses and therefore additional markers of inflammation are needed. So far, only a limited number of longitudinal studies have used a combination of symptoms and additional measurements [e.g. calprotectin or endoscopy] to define flares. ${ }^{11,13,14,26}$ In a longitudinal study using a combination of clinical disease activity index and faecal calprotectin concentration, short-term perceived stress was positively associated with subsequent IBD symptoms, but not with 'objectively' assessed inflammation by faecal calprotectin measurement. ${ }^{13}$ These findings may explain some contradictory results from earlier studies and support our findings concerning the absence of an association between the presence of perceived stress in general and flares. Nonetheless, a difference between the presence of perceived stress in general and novel perceived stress was observed here. In many studies, 'shortterm' perceived stress has been analysed by using a questionnaire comprising the past month [e.g. Cohen Perceived Stress Scale ${ }^{24}$ or Perceived Stress Questionnaire $\left.{ }^{27}\right]$. Unfortunately, these questionnaires are insensitive to perceived stress in the period prior to this month. Therefore, exposure time to perceived stress is unknown and both 'novel' and 'longer lasting' stress are measured. To analyse the effect of intra-individual changes of perceived stress over time, longitudinal follow-up of both the outcome variable [i.e. disease flares] and perceived stress is necessary. To our knowledge, in only a few studies have both variables been repeatedly measured during follow-up. ${ }^{9-13,26}$ Of these, none used the repetitive assessments to differentiate novel perceived stress from chronic or pre-existing perceived stress.

In the present study, we observed a clear difference between the effect of perceived stress in general and the effect of novel perceived stress. In healthy individuals, both internal and external stressors are in balance with behavioral and physiological adaptive responses. Perceived stress may become detrimental when this balance is disturbed [e.g. via acute stressors]. ${ }^{28}$ It is hypothesized that perceived stress may affect the brain-gut axis through: [1] activation of the hypothalamic-pituitary-adrenal axis and subsequent release of corticotropin-releasing factor and cortisol, which have direct and indirect gastrointestinal immunostimulatory effects; [2] decreased activity of the vagal nerve and thereby a decreased anti-inflammatory effect; [3] increased activation of the sympathetic nervous system and subsequent increase of inflammatory cytokines, activation of the NF-kB pathway, and activation of mast cells; and [4] peripheral release of substance $P$ by the enteric nervous system, acting as an inflammatory cytokine and stimulator of mast cells. ${ }^{6,16}$ It is hypothesized that after such disturbance, homeostasis usually returns in most individuals. ${ }^{28}$ Therefore, we suggest that during prolonged stress, individuals can adapt to this new situation. This might explain why, in our study, only novel perceived stress was associated with flares. However, another longitudinal study observed long-term perceived stress [i.e. covering 2 years prior to measurement] to be associated with flares in the following 8 months, while short-term perceived stress [i.e. covering the month prior to measurement] was not. ${ }^{26}$ However, their explanation of a proposed lengthy time scale for the evolution of effects of stress on the intestines was primarily based on animal studies. Coping strategies and consequent adaptation to chronic stress may be different in humans. It is hypothesized that chronic stress enhances the effects of acute stress through greater and more prolonged increases in sympathetic activation following a stressor, although supporting evidence for this is scarce. ${ }^{16}$ Future studies regarding perceived stress and disease flares, using the same methodology as the present study, should be performed to validate our findings.

The occurrence of life events was also associated with the development of a flare in the next 3 months. The most frequently identified type of life events were work-related [19.4\%; e.g. dismissal or reorganization], personal health issues [10.9\%], moving house or renovating a house $[10.9 \%$ ], or the death of relatives or good friends [9.1\%]. The effect of life events on future disease activity is smaller when compared to perceived stress. Although life events by definition are correlated with perceived stress, the way of coping will determine whether the life event as a stressor leads to more perceived stress or not. We speculate that only patients with insufficient coping strategies will experience an increase in perceived stress. Unfortunately, our study is not designed to analyse these effects. However, this hypothesis is supported by findings of another prospective study in which a combination of low stress and a good coping strategy was associated with the lowest risk of a disease flare. ${ }^{12}$ Debate remains as contradictory results on the influence of life events have been reported by others, possibly due to differences in study design and methodology. ${ }^{8,29}$

In this study, no association was found between anxiety, depression or fatigue and subsequent disease flares. Anxiety and depression as comorbidities have extensively been studied in IBD patients and in a recent systematic review it has been shown that the prevalence rate of anxiety [20\%] and depression [15\%] is high in IBD compared to prevalence rates in the general population $[7.3 \%$ and $3.2 \%$, respectively]. ${ }^{5,30,31}$ However, a meta-analysis of prospective studies on the impact of a depressive state on disease activity showed no statistically significant association, which is in line with our study. ${ }^{32}$ The association between both anxiety and disease flares as well as fatigue and disease flares has not very frequently been studied. Anxiety seemed not to be associated, as in our study, with disease flares. ${ }^{11,12,33}$ Although longitudinal evidence for an association between fatigue and flares is lacking, a high prevalence of fatigue in active IBD has been observed. ${ }^{34}$ In our study, this association could not be confirmed.

The strength of this study is the systematic and continuous monitoring of both disease activity and psychosocial wellbeing over a 12 -month period in a large study population. Additionally, we were able to analyse within-subject changes of psychosocial wellbeing. Also, we defined disease flares using a combination of clinical disease activity [i.e. MIAH-questionnaire] and additional parameters [i.e. faecal calprotectin, imaging or outpatient clinic visit with treatment adjustment]. Some limitations also need to be addressed. First and most importantly, we did not use validated questionnaires for psychosocial wellbeing in the telemedicine tool. The questionnaires in myIBDcoach were designed to screen for several relevant aspects potentially 
associated with patients' wellbeing and these questionnaires had to be concise for repeated use in a daily clinical practice setting. We therefore chose to apply only one 'screening' question per domain to limit loss of adherence. However, previous studies have shown that single-question questionnaires can correlate well with comprehensive questionnaires [e.g. for perceived stress ${ }^{35}$ ]. Second, we were not able to obtain reliable data on the use of non-steroidal anti-inflammatory drugs, the use of antibiotics or incident infections from the myIBDcoach tool, whereas several studies suggest that these variables are associated with disease flares. ${ }^{29}$ On the other hand, a large longitudinal population-based study showed no effect of either of these variables and therefore we believe that the potential confounding effect, if present, is only minimal. ${ }^{9}$

Currently, we believe that the merit of psychosocial wellbeing in IBD patients is underestimated and hardly routinely assessed in daily practice. In this study, we addressed that particular attention should be paid to novel perceived stress and life events and our data underline a possible need for tight psychosocial monitoring of IBD patients. Our findings concerning the association between novel perceived stress and disease flares need confirmation by future studies and such studies should take coping strategies into account. Eventually, personalized interventions in those who are at risk of developing flares are worthy of further study.

In conclusion, the occurrence of recent life events and novel perceived stress were prospectively associated with the incidence of IBD disease flares in the next 3 months, while the presence of perceived stress in general was not. Also, no association was found between disease flares and anxiety, depression or fatigue. This study underlines the importance of continuous personalized monitoring of IBD patients, which may contribute to the prevention of disease flares.

\section{Funding}

This work was supported by an academic incentive fund of the MUMC+ [31962340B].

\section{Conflict of Interest}

The authors have no conflicts of interest regarding this manuscript.

\section{Acknowledgments}

M. Cilissen, W. Hameeteman, T. van den Heuvel, R. Huibregtse, S. Jeuring, S. Pessers, D. Roosen, C. Spooren, H. Tomlow, B. Verhaegh, W. van de Wetering, A. Wolters, N. Ipenburg, N. Peek, H. Slingerland, R. Veenendaal, M. Verwey, L. Colautti-Duijsens, E. Keulen, J. Wilbrink, M. van Kouwen, M. Somers, K. Achterberg, E. Smit, M. Spruit.

\section{Author Contributions}

MJP and MJJ conceived the study and developed the myIBDcoach telemedicine tool. DSJW, MJJ, AEvdMJ, MJRC, MCB, JPM, AAvB, NM and MJP collected the data. DSJW, BW, DMAEJ and MJP analysed and interpreted the data. DSJW and MJP drafted the manuscript. MJJ, AEvdMJ, MJRC, MCB, JPM, AAvB, NM, TM, JH, AAMM, BW and DMAEJ critically reviewed the data and first drafts. All authors critically reviewed and approved the final manuscript. MJP is guarantor of this article.

\section{References}

1. Torres J, Mehandru S, Colombel JF, Peyrin-Biroulet L. Crohn's disease. Lancet 2017;389:1741-55.

2. Ungaro R, Mehandru S, Allen PB, Peyrin-Biroulet L, Colombel JF. Ulcerative colitis. Lancet 2017;389:1756-70.
3. Yarur AJ, Strobel SG, Deshpande AR, Abreu MT. Predictors of aggressive inflammatory bowel disease. Gastroenterol Hepatol 2011;7:652-9.

4. Walker JR, Ediger JP, Graff LA, et al. The Manitoba IBD cohort study: a population-based study of the prevalence of lifetime and 12-month anxiety and mood disorders. Am J Gastroenterol 2008;103:1989-97.

5. Neuendorf R, Harding A, Stello N, Hanes D, Wahbeh H. Depression and anxiety in patients with inflammatory bowel disease: a systematic review. J Psychosom Res 2016;87:70-80.

6. Bonaz BL, Bernstein CN. Brain-gut interactions in inflammatory bowel disease. Gastroenterology 2013;144:36-49.

7. Martin-Subero M, Anderson G, Kanchanatawan B, Berk M, Maes M. Comorbidity between depression and inflammatory bowel disease explained by immune-inflammatory, oxidative, and nitrosative stress; tryptophan catabolite; and gut-brain pathways. CNS Spectr 2016;21:184-98.

8. Triantafillidis JK, Merikas E, Gikas A. Psychological factors and stress in inflammatory bowel disease. Expert Rev Gastroenterol Hepatol 2013;7:225-38.

9. Bernstein CN, Singh S, Graff LA, Walker JR, Miller N, Cheang M. A prospective population-based study of triggers of symptomatic flares in IBD. Am J Gastroenterol 2010;105:1994-2002.

10. Langhorst J, Hofstetter A, Wolfe F, Häuser W. Short-term stress, but not mucosal healing nor depression was predictive for the risk of relapse in patients with ulcerative colitis: a prospective 12-month follow-up study. Inflamm Bowel Dis 2013;19:2380-6.

11. Bitton A, Sewitch MJ, Peppercorn MA, et al. Psychosocial determinants of relapse in ulcerative colitis: a longitudinal study. Am J Gastroenterol 2003;98:2203-8.

12. Bitton A, Dobkin PL, Edwardes MD, et al. Predicting relapse in Crohn's disease: a biopsychosocial model. Gut 2008;57:1386-92.

13. Sexton KA, Walker JR, Graff LA, et al. Evidence of bidirectional associations between perceived stress and symptom activity: a prospective longitudinal investigation in inflammatory bowel disease. Inflamm Bowel Dis 2017;23:473-83.

14. Cámara RJ, Schoepfer AM, Pittet V, Begré S, von Känel R; Swiss Inflammatory Bowel Disease Cohort Study [SIBDCS] Group. Mood and nonmood components of perceived stress and exacerbation of Crohn's disease. Inflamm Bowel Dis 2011;17:2358-65.

15. Gracie DJ, Williams CJ, Sood R, et al. Poor correlation between clinical disease activity and mucosal inflammation, and the role of psychological comorbidity, in inflammatory bowel disease. Am J Gastroenterol 2016;111:541-51.

16. Mawdsley JE, Rampton DS. Psychological stress in IBD: new insights into pathogenic and therapeutic implications. Gut 2005;54:1481-91.

17. Agostini A, Ballotta D, Righi S, et al. Stress and brain functional changes in patients with Crohn's disease: a functional magnetic resonance imaging study. Neurogastroenterol Motil 2017;29:1-10.

18. Trindade IA, Ferreira C, Moura-Ramos M, Pinto-Gouveia J. An 18-month study of the effects of IBD symptomatology and emotion regulation on depressed mood. Int J Colorectal Dis 2017;32:651-60.

19. de Jong MJ, van der Meulen-de Jong AE, Romberg-Camps MJ, et al. Telemedicine for management of inflammatory bowel disease (myIBDcoach): a pragmatic, multicentre, randomised controlled trial. Lancet 2017;390:959-68.

20. de Jong $\mathrm{M}$, van der Meulen-de Jong A, Romberg-Camps $\mathrm{M}$, et al. Development and feasibility study of a telemedicine tool for all patients with IBD: MyIBDcoach. Inflamm Bowel Dis 2017;23:485-93.

21. de Jong M, Van den Heuvel T, Romberg-Camps M, Winkens B, Markus $\mathrm{T}$, Masclee A, et al. Development of a patient reported disease activity score to screen for mucosal inflammation in inflammatory bowel disease. J Crohns Colitis 2015;9(Suppl 1): S192-3.

22. Zigmond AS, Snaith RP. The hospital anxiety and depression scale. Acta Psychiatr Scand 1983;67:361-70.

23. Bleijenberg G KH, Gielissen M. De Verkorte VermoeidheidsVragenlijst voor het vaststellen van de ernst van chronische vermoeidheid. Bijblijven 2009;25:19-21.

24. Cohen S, Kamarck T, Mermelstein R. A global measure of perceived stress. J Health Soc Behav 1983;24:385-96. 
25. Keefer L, Keshavarzian A, Mutlu E. Reconsidering the methodology of "stress" research in inflammatory bowel disease. J Crohns Colitis 2008;2:193-201.

26. Levenstein S, Prantera C, Varvo V, et al. Stress and exacerbation in ulcerative colitis: a prospective study of patients enrolled in remission. Am J Gastroenterol 2000;95:1213-20.

27. Levenstein S, Prantera C, Varvo V, et al. Development of the Perceived Stress Questionnaire: a new tool for psychosomatic research. J Psychosom Res 1993;37:19-32.

28. Chrousos GP. Stress and disorders of the stress system. Nat Rev Endocrinol 2009;5:374-81.

29. Singh S, Graff LA, Bernstein CN. Do NSAIDs, antibiotics, infections, or stress trigger flares in IBD? Am J Gastroenterol 2009;104:1298-313; quiz 1314.

30. Baxter AJ, Scott KM, Vos T, Whiteford HA. Global prevalence of anxiety disorders: a systematic review and meta-regression. Psychol Med 2013;43:897-910.
31. Moussavi S, Chatterji S, Verdes E, Tandon A, Patel V, Ustun B. Depression, chronic diseases, and decrements in health: results from the World Health Surveys. Lancet 2007;370:851-8.

32. Alexakis C, Kumar S, Saxena S, Pollok R. Systematic review and metaanalysis: the impact of a depressive state on disease course in adult inflammatory bowel disease. Aliment Pharmacol Ther 2017;46:225-35.

33. Dhingra R, Kedia S, Mouli VP, et al. Evaluating clinical, dietary, and psychological risk factors for relapse of ulcerative colitis in clinical, endoscopic, and histological remission. J Gastroenterol Hepatol 2017;32:1698-705.

34. Huppertz-Hauss G, Høivik ML, Jelsness-Jørgensen LP, et al. Fatigue in a population-based cohort of patients with inflammatory bowel disease 20 years after diagnosis: the IBSEN study. Scand J Gastroenterol 2017;52:351-8.

35. Littman AJ, White E, Satia JA, Bowen DJ, Kristal AR. Reliability and validity of 2 single-item measures of psychosocial stress. Epidemiology 2006;17:398-403.

36. Satsangi J, Silverberg MS, Vermeire S, Colombel JF. The Montreal classification of inflammatory bowel disease: controversies, consensus, and implications. Gut 2006;55:749-53. 